\title{
Age Effects in Cultural Life Scripts
}

\author{
STEVE M. J. JANSSEN ${ }^{1,2 *}$ and DAVID C. RUBIN ${ }^{1}$ \\ ${ }^{1}$ Department of Psychology and Neuroscience, Duke University, Durham, USA \\ ${ }^{2}$ Department of Psychology, University of Amsterdam, Amsterdam, the Netherlands
}

\begin{abstract}
Summary: Life scripts are culturally shared expectations about the timing of life events in an idealized life course. Because they are cultural semantic knowledge, they should be known by all adult age groups including those who have not lived through all events in the life script, but this has not been tested previously. Young, middle-aged and older adults from the Netherlands were therefore asked in this online study to imagine an ordinary Dutch infant and to name the seven most important events that were likely to take place in the life of this prototypical child. Participants subsequently answered questions about at what ages these events were expected to occur and about their prevalence, importance and valence. We found that the cultural life script was similar for young, middle-aged and older adults and for adults with different educational attainment. Copyright (C) 2010 John Wiley \& Sons, Ltd.
\end{abstract}

When one examines the temporal distribution of autobiographical memory, one can identify three components. People hardly recall any personal events from the first 3 or 4 years of their lives, but many events from the most recent years. The prior effect is called childhood amnesia, while the latter is called the recency effect. Between these two components, there is the reminiscence bump, which is the effect that people recall more personal events from adolescence and early adulthood than from adjacent lifetime periods (Rubin, Rahhal, \& Poon, 1998; Rubin, Wetzler, \& Nebes, 1986; Rybash, 1999).

The concept of a life script was initially formulated based on the unexpected finding that the request of events in which people had one of several extremely positive emotions produced a reminiscence bump, but requests of events cued by extremely negative events did not (Berntsen \& Rubin, 2002; Rubin \& Berntsen, 2003). This fits with an existing literature in sociology on the concept of cultural age norms (Neugarten, Moore, \& Lowe, 1965), noting that many important events are expected to occur at a given age and sequence. Moreover, it was noted that in most cultures there are no emotionally negative events with known expected dates and sequencing (Berntsen \& Rubin, 2002; Rubin \& Berntsen, 2003). The time locked nature of the events and their positive affect led to modification of the script concept from cognitive psychology (Schank \& Abelson, 1977).

Berntsen and Rubin (2004) examined the cultural life script by asking undergraduates to imagine an ordinary infant and to name the seven most important events that were likely to take place in the life of this prototypical newborn baby. Participants then indicated at what age the events were most likely to occur and to rate the events on prevalence, importance and valence. The reported events were mainly normative life events (e.g. graduation, marriage and retirement) and the majority of the events was positive,

*Correspondence to: Steve M. J. Janssen, Steve Janssen, Department of Psychology, Hokkaido University, Kita-10 Nishi-7 Kita-ku, Sapporo, 0600810, Japan. E-mail: janssen@let.hokudai.ac.jp which was consistent with the property that life scripts represent an idealized life. The standard deviations of the expected age of occurrence of the positive events were smaller than the standard deviations of the expected age of the negative events. Most positive events were expected to occur during early adulthood, whereas the negative events could happen at any time in life. The results were consistent with the notions that the life scripts favour positive events and events from early adulthood. Since the participants nominated many events that they had probably not experienced yet, like retirement, getting grandchildren, the death of a partner or own death, the results of the study could thus not be attributed to the participants' own experiences of these life events. The knowledge about these life events was already present. These findings were then confirmed and extended by many additional studies (Berntsen \& Jacobsen, 2008; Bohn \& Berntsen, 2008; Collins, Pillemer, Ivcevic, \& Gooze, 2007; Erdoğan, Baran, Avlar, Taş, \& Tekcan, 2008; Glück \& Bluck, 2007; Habermas, 2007, Study 2; Rubin, Berntsen, \& Hutson, 2009; Thomsen \& Berntsen, 2008).

From such studies it has been found that cultural life scripts have the following 10 properties, of which the first six are derived from Schank and Abelson's (1977) script notion, whereas the four remaining properties differ from their notion (Berntsen \& Rubin, 2004; Rubin et al., 2009). (1) Life scripts are semantic knowledge about expectations about life events in a given culture and not personal memories of those life events. (2) Life scripts are a series of temporally ordered life events. (3) Life scripts form a hierarchical arrangement with transitional events forming a higher order 'scene', in which a series of subordinate actions or episodes are nested. (4) Life scripts can be described in terms of time slots and their requirements. (5) The time slots are culturally important transitional events and their requirements are the culturally sanctioned timing of these events. (6) Life scripts are used to process life stories. (7) Life scripts do not represent an average life, but represent an idealized life from which many common and some important events are left out. (8) Because life scripts represent a normative life course, life scripts are not extracted from personal actions in recurrent 
contexts, but are transmitted by tradition. Young people, who have lived through a smaller part of their life, still know the entire life script of their culture. (9) Life scripts are distorted from actual lives to favour positive events. (10) Life scripts are distorted from actual life stories to favour events expected to occur in the early adulthood.

Erdoğan et al. (2008) altered Berntsen and Rubin's (2004) design slightly. They asked university students to name the seven most important personal events that a newborn baby would experience in his or her life or the seven events that an elderly person would have experienced in his or her life. The reported events of the two groups were similar. Seven events were among the 10 most named events in both groups. Both groups also named more positive events than negative events. The positive events came mostly from the third decade of the infant's or the elderly person's life, while the negative events were in both groups equally distributed across the life span. While both groups named most events from the third decade, the participants named relatively more events from the first two decades for the newborn baby, whereas the other participants named relatively more events from the period in which the elderly person would have been between 30 and 70 years old.

There are several studies which have shown that people use the cultural life script to process their life stories. There is a large overlap between the life scripts and life stories of young adults (Rubin et al., 2009) and middle-aged adults (Collins et al., 2007), and there is an even larger overlap between the life scripts and life stories of older adults (Glück \& Bluck, 2007; Thomsen \& Berntsen, 2008).

Cultural life scripts also seem to be a pre-requisite for the development of life stories. Although the ability to remember personal events emerges across the preschool years (Fivush \& Nelson, 2004), children only start to develop a coherent life story in adolescence (Habermas \& Bluck, 2000). Habermas and Bluck distinguished four types of coherence in life narratives: Temporal, thematic and causal and biographical coherence. Temporal coherence emerges around the age of 9 years, while thematic coherence appears in middle adolescence. Causal and biographical coherence emerge in middle to late adolescence. Bohn and Berntsen (2008) asked children who attended third, fifth and sixth, or eight grade to recall a recent personal event, their life story and the cultural life script. A group of young adults were also asked to provide the life script. Bohn and Berntsen rated the single event and the life story on global coherence, while the life script of the young adults was used to establish the typicality of the children's life script. Bohn and Berntsen found that third-graders wrote more coherent reports about the single events than about their life story, but the coherence of the single event and the life story both increased with age. Furthermore, the life scripts became more similar to the norms provided by the young adults as the children became older. Bohn and Berntsen found, surprisingly, no correlation between the coherence of the single event and the life story when they controlled for age. There was, however, a strong correlation between typicality of the life script and the coherence of the life story. Children, who named 10 events in the life script study that were often named by the young adults, also told coherent life stories.
One key assumption, and the one we focus on here, remains unstudied: Whether life scripts are really learned from cultural transmission rather than from living a life and generalizing from that life. This claim is an essential part of the concept of cultural life scripts for three reasons. First, the basic idea of life scripts is that they are shared, semantic knowledge rather than autobiographical knowledge. They are supposed to help structure autobiographical memory, not be a product of it. Second, because cultural life script events are more positive than the average of many lived lives, they should represent the normative life as it is supposed to be lived, not a generalization from a life lived. Third, if cultural life scripts are supposed to guide understanding and help structure the recall of autobiographical events and life stories, then the earlier they are mastered, the more useful they will be. Ideally that should be as young as the developmental studies cited report that it is possible to have an overview of a life narrative.

Therefore, in the present study, participants who varied in age from young adulthood to older adulthood provided a life script by naming the seven most important events that are most likely to take place in the life of a prototypical infant. This allowed us to examine the life script for age effects. The absence of age differences would support the view that cultural life scripts are semantic memory rather than instance-based learning. Young adults are, therefore, expected to nominate the same events as middle-aged and older adults. Small differences in the life scripts could be tolerated by the theory if they were caused by cohort effect that represented genuine changes in cultural views, but these should be minimal. Another question the study allows us to ask is whether the life script obtained holds for the culture as a whole or whether it changes with education. This is interesting because many of the life script events change with education, either in their occurrence or absence or in their time of occurrence. For instance, obtaining a university degree often delays marriage and the birth of children. If the life script is the same for an entire culture and not just a generalization from one's own experiences, these differences should also be minimal.

This study is the first one, in which middle-aged and older adults were asked to imagine a newborn infant and to generate seven highly important events that will likely take place in this infant's life (although Bohn, in press). Habermas (2007, Study 2) asked 213 participants, of whom 81 were between 26 and 75 years old, to produce the life script, he only analysed the cultural life script of participants who were 20 years old. In other studies (Collins et al., 2007; Glück \& Bluck, 2007; Thomsen \& Berntsen, 2008), middle-aged and older adults were only asked to report the most important events from their own lives.

Like previous studies (Berntsen \& Rubin, 2004; Erdoğan et al., 2008; Habermas, 2007, Study 2; Rubin et al., 2009), we expect to find that the standard deviations of the expected ages are smaller for positive events than for negative events, suggesting that positive events are likely to occur in a certain age frame, while negative events can occur at any moment. Furthermore, we expect to find that the positive events mostly come from the third decade, while the negative and neutral events are expected to be distributed equally across the life span. 


\section{METHOD}

\section{Participants}

The life script questionnaire was administered via the Internet at http://memory.uva.nl. Participants could come into contact with the website in at least four ways: (1) through links on other websites, (2) through search engines, (3) through promotion in traditional media, such as newspapers and magazines, and (4) through word of mouth. At the end of the questionnaire, participants could invite friends, relatives or colleagues to take part in the study as well by sending them standardized e-mails. Furthermore, participants who had made other tests on our website, such as the Favourites Questionnaire (Janssen, Chessa, \& Murre, 2007) or the Yearly News Memory Test (Janssen, Murre, \& Meeter, 2008), were invited to take part in this study as well. The participants were not financially or otherwise compensated for their participation.

There were 595 Dutch participants who completed the study. Nearly all participants were female (90.4\%). The average age of the participants was 44.3 years $(S D=14.7)$. They were divided into three age groups of equal length (i.e., 20 years). There were 214 participants between the ages of 16 and 35, 258 between 36 and 55 and 123 between 56 and 75 .

The participants were also divided into four levels within the Dutch educational system. Lower level consists of participants $(N=47)$ who finished primary school ('lagere school', 8 years of education) or lower professional education ('LBO', 12 years). Participants in the middle level $(N=150)$ completed either middle general secondary education ('MAVO', 12 years) or middle professional education ('MBO', 16 years). Higher level consists of participants $(N=238)$ who completed higher general secondary education ('HAVO', 13 years) or higher professional education ('HBO', 17 years). Participants with scientific level $(N=160)$ finished preparatory scientific education ('VWO', 14 years) or scientific education ('WO', 18 years). The Dutch system cannot be fully explained in terms of years of education, since the levels often run parallel from each other. Lower levels are generally more focused on vocation, while higher levels are more academically orientated.

\section{Procedure}

At the beginning of the study, participants were asked to imagine an ordinary infant that had the same gender as themselves. The infant could not be a specific child that the participants knew, but he or she had to be a prototypical infant from the Netherlands with an ordinary life course ahead. The participants were then asked to describe the seven most important events that were likely to take place in the life of this prototypical infant.

After the participants had entered seven events, they were given the descriptions of the events back one by one. For each event, they are asked if the event could be categorized into one of 46 options that were presented in a drop-down menu. The options consisted of descriptions derived from Berntsen and Rubin (2004), Erdoğan et al. (2008) and Rubin et al. (2009) and were presented in alphabetic order (and not on age of occurrence or topic). If there was no description that suited the event, the participants were asked to select the last option, called 'other'. The list of options is given in Table 1 . Confirmation is missing from this list, because no participant selected this event.

Participants were then asked to indicate on a drop-down menu that ranged from 0 to 100 people how many people would experience the event at least once in their lifetime, to rate how important the event was on a seven-point scale that ranged from 'unimportant' (1) to 'greatest importance' (7), to indicate on a drop-down menu that ranged from 0 to 100 years at what age the event would most likely occur, to rate the pleasantness of the event on a seven-point scale that ranged from 'very negative' ( -3$)$ to 'very positive' (3), and to indicate which emotion a person would probably feel when experiencing this event. Participants could select one emotion from 'happiness', 'anger', 'in love', 'fear', 'sadness', 'pride' and 'jealousy' or they could enter an alternative emotion in an open text field.

At the end of the questionnaire, participants were thanked for their contribution and were briefly told about the background of the study. They were given the opportunity to ask us questions and to send friends, family and colleagues a standardized invitation.

\section{RESULTS}

Given the considerations associated with Internet testing (e.g., Gosling, Vazire, Srivastava, \& John, 2004; Reips, 2000, 2002), four measures were taken to ensure quality control. (1) The analyses were restricted to the results of Dutch participants who were between 16 and 75 years old. (2) Participants could take the questionnaire as often as they wanted, but only their first contribution was included. (3) Questionnaires that were not completed were omitted. (4) Some participants nominated six or seven events that were expected to occur before the age of 10 years. The results of these participants $(N=29)$ were excluded from the analyses as well. The questionnaires of the previously mentioned 595 participants met these four criteria.

After participants had nominated seven events, they indicated at which age they expected the event to occur and they rated the prevalence, importance and valence of each event. Since participants were asked for the most important events that people would most likely experience, it is not surprising that the events were rated as being very important $(M=5.94, S D=1.04)$ and that most people were likely to experience the events $(M=80.1, S D=22.2)$.

Consistent with the properties of life scripts listed earlier, the majority of the events was positive, $\chi^{2}(1, N=4165)=$ 904.56, $p<.001$, and the distribution of the positive events $(73.3 \%)$ was heavily tilted towards early adulthood and early childhood. More than half of the positive events $(55.4 \%)$ were expected to occur between the ages of 16 and 30 and a fifth of the positive events $(20.0 \%)$ were expected to happen in the first 6 years. The participants hardly nominated any positive events that were expected to take place after the age of $30(8.4 \%)$. The remaining positive events $(16.2 \%)$ were expected to occur when the imaginary person was between 6 and 15 years old. The negative and neutral 
Table 1. The descriptions of the categories, including the number of mentions, the frequency of mentions, the average expected age, the standard deviation of expected age, the average prevalence, the average importance and the average valence

\begin{tabular}{|c|c|c|c|c|c|c|c|}
\hline Description & $\begin{array}{l}\text { Number of } \\
\text { mentions }\end{array}$ & $\begin{array}{c}\text { Frequency } \\
(\%)\end{array}$ & $\begin{array}{l}\text { Mean } \\
\text { age }\end{array}$ & $\begin{array}{l}\text { SD } \\
\text { age }\end{array}$ & $\begin{array}{c}\text { Mean } \\
\text { prevalence }\end{array}$ & $\begin{array}{c}\text { Mean } \\
\text { importance }\end{array}$ & $\begin{array}{c}\text { Mean } \\
\text { valence }\end{array}$ \\
\hline Having children & 429 & 72.1 & 28.92 & 2.55 & 67.72 & 6.48 & 2.60 \\
\hline Begin school & 360 & 60.5 & 4.53 & 0.90 & 96.76 & 5.91 & 1.65 \\
\hline Marriage & 316 & 53.1 & 27.13 & 3.69 & 67.12 & 5.87 & 2.35 \\
\hline Parents' death & 259 & 43.5 & 49.72 & 9.28 & 91.52 & 6.30 & -1.97 \\
\hline Fall in love & 243 & 40.8 & 13.79 & 2.63 & 94.42 & 5.81 & 2.00 \\
\hline First fulltime job & 193 & 32.4 & 22.19 & 2.52 & 85.34 & 5.87 & 1.93 \\
\hline Leave home & 113 & 19.0 & 20.58 & 2.36 & 90.65 & 5.93 & 1.73 \\
\hline High school & 108 & 18.2 & 12.15 & 0.64 & 90.95 & 5.81 & 1.44 \\
\hline High school graduation & 99 & 16.6 & 17.52 & 0.91 & 78.58 & 5.80 & 2.27 \\
\hline Meeting spouse & 99 & 16.6 & 23.79 & 3.83 & 78.40 & 6.05 & 2.28 \\
\hline Own death & 99 & 16.6 & 80.80 & 6.77 & 100.00 & 6.17 & -0.94 \\
\hline College graduation & 98 & 16.5 & 23.31 & 1.87 & 57.21 & 5.94 & 2.57 \\
\hline Serious illness & 88 & 14.8 & 50.11 & 17.00 & 58.56 & 6.19 & -2.00 \\
\hline Learning to walk & 87 & 14.6 & 1.22 & 0.65 & 96.87 & 6.47 & 2.86 \\
\hline First sexual experience & 79 & 13.3 & 16.34 & 1.88 & 93.78 & 6.08 & 1.65 \\
\hline Adolescence/Puberty & 76 & 12.8 & 13.42 & 2.00 & 89.80 & 5.89 & 0.82 \\
\hline Grandparents' death & 64 & 10.8 & 19.73 & 8.56 & 88.66 & 5.67 & -1.81 \\
\hline Attending college & 60 & 10.1 & 18.37 & 1.57 & 49.62 & 5.72 & 1.95 \\
\hline Making long journey & 52 & 8.7 & 22.12 & 6.75 & 57.98 & 4.98 & 2.04 \\
\hline Divorce & 51 & 8.6 & 38.49 & 5.60 & 47.02 & 6.08 & -1.86 \\
\hline Grandchildren & 50 & 8.4 & 58.38 & 5.15 & 60.72 & 5.84 & 2.40 \\
\hline Retirement & 50 & 8.4 & 65.66 & 5.77 & 79.56 & 5.54 & 0.86 \\
\hline Settle on career & 49 & 8.2 & 22.45 & 4.91 & 76.31 & 5.94 & 1.61 \\
\hline Birth sibling & 47 & 7.9 & 2.74 & 0.67 & 70.43 & 5.60 & 1.34 \\
\hline Find right job & 46 & 7.7 & 25.78 & 4.41 & 74.54 & 5.91 & 1.80 \\
\hline Buy house & 46 & 7.7 & 27.70 & 3.92 & 63.00 & 5.43 & 1.76 \\
\hline Learning to swim or cycle ${ }^{a}$ & 44 & 7.4 & 5.52 & 1.64 & 85.75 & 5.20 & 1.89 \\
\hline Death friend & 43 & 7.2 & 35.72 & 16.92 & 78.63 & 6.05 & -1.81 \\
\hline Moving & 41 & 6.9 & 19.49 & 8.28 & 82.68 & 4.90 & 0.80 \\
\hline First play friend & 39 & 6.6 & 3.82 & 1.80 & 91.62 & 6.38 & 2.33 \\
\hline Learning to talk & 38 & 6.4 & 1.29 & 0.68 & 97.00 & 6.82 & 2.97 \\
\hline Death spouse & 36 & 6.1 & 67.86 & 12.28 & 63.67 & 6.61 & -2.33 \\
\hline Group of friends & 34 & 5.7 & 16.44 & 4.49 & 84.56 & 6.15 & 1.97 \\
\hline Own birth & 33 & 5.5 & 0.00 & 0.00 & 100.00 & 6.88 & 1.94 \\
\hline Daycare & 32 & 5.4 & 2.41 & 1.20 & 84.88 & 5.47 & 1.09 \\
\hline Driver's license & 32 & 5.4 & 19.22 & 0.89 & 79.88 & 5.25 & 2.00 \\
\hline First rejection & 29 & 4.9 & 14.59 & 4.23 & 84.10 & 5.41 & -1.52 \\
\hline Accident & 23 & 3.9 & 22.87 & 12.14 & 60.35 & 4.65 & -1.65 \\
\hline Adulthood & 21 & 3.5 & 20.76 & 6.02 & 86.38 & 6.00 & 1.71 \\
\hline Moving in with somebody ${ }^{a}$ & 21 & 3.5 & 23.38 & 2.38 & 78.86 & 5.81 & 2.00 \\
\hline Divorce parents ${ }^{\mathrm{a}}$ & 20 & 3.4 & 13.05 & 5.82 & 49.80 & 5.85 & -1.85 \\
\hline First part-time job & 20 & 3.4 & 15.55 & 0.86 & 78.95 & 4.85 & 1.55 \\
\hline Major achievement & 20 & 3.4 & 23.25 & 12.42 & 57.80 & 5.95 & 2.10 \\
\hline Children leave home & 13 & 2.2 & 52.15 & 4.13 & 65.62 & 5.69 & 1.00 \\
\hline Important promotion & 11 & 1.8 & 33.64 & 7.10 & 45.18 & 5.55 & 1.82 \\
\hline Problems work ${ }^{\mathrm{a}}$ & 10 & 1.7 & 30.60 & 9.83 & 54.80 & 5.60 & -2.00 \\
\hline First holy communion & 5 & 0.8 & 7.80 & 0.40 & 37.60 & 5.80 & 2.20 \\
\hline Baptism & 4 & 0.7 & 0.50 & 0.50 & 45.00 & 5.25 & 2.25 \\
\hline Other & 335 & 56.3 & 14.67 & 19.02 & 81.47 & 5.60 & 1.04 \\
\hline
\end{tabular}



events $(26.7 \%)$ were more equally distributed. About a fifth of these events $(19.2 \%)$ were expected to occur in early adulthood (16-30 yrs). The participants also nominated relatively many negative events $(11.5 \%)$ that were expected to occur in early childhood ( $0-5$ years). Almost half of the negative and neutral events $(51.7 \%)$ were expected to take place in middle and late adulthood $(31+$ years $)$. The remaining negative events $(17.5 \%)$ were expected to occur when the imaginary person would be between 6 and 15 years old. Figure 1 shows the proportion of positive and negative events as a function of their expected age at occurrence.

Because the positive and negative events are distributed differently, events that were expected to occur before the age of 36 are on average positive $(M=1.75, S D=1.47$, $t(3474)=69.17, p<.001)$, while the events that were expected to occur after the age of 35 are generally negative $(M=-0.99, \quad S D=1.90, \quad t(689)=13.60, \quad p<.001)$. The average valence of the nominated events as a function of their expected age at occurrence is shown in Figure 2. 


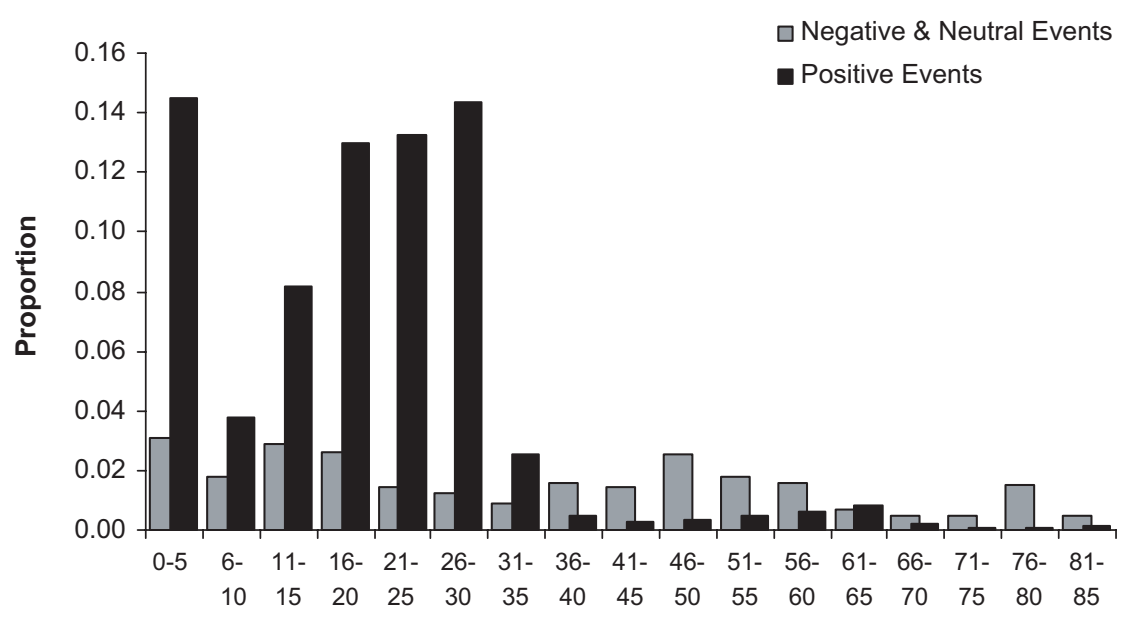

Lifetime Period

Figure 1. The proportion of positive and negative events as a function of their expected age at occurrence

\section{Categories}

Participants were asked to categorize the events. In Table 1, the number and percentage of mentions, the mean values of the ratings scales and the standard deviation of the expected age of occurrence per category are given. The participants classified about $10 \%$ of the events as 'other'. Four extra categories could be established on the basis of the descriptions: Learning to swim, cycle or play a musical instrument; divorce of parents; moving in with somebody; and problems at work. The remaining descriptions were very infrequent (e.g., 'first birthday', 'learning to read and write', 'menopause', 'depression'). There were 37 categories of events that were nominated by at least $4 \%$ of the sample. Remarkably, categories about religious events were hardly used, while events, such as confirmation and circumcision, were a part of the Danish and Turkish life script (Berntsen \& Rubin, 2004; Erdoğan et al., 2008).

Consistent with the properties of life scripts listed earlier, most categories that were named by at least $4 \%$ of the sample were positive (29 positive and 8 negative categories, $\chi^{2}(1$, $N=37)=11.92, p=.001$, under the assumption that the categories are independent units). Positive categories were not more important $(M=5.86, S D=0.47)$ than negative categories $(M=6.06, S D=0.37 ; t(35)=1.11, p=.275)$. Positive categories $(M=80.2, S D=13.7)$ were also not more prevalent than negative categories $(M=76.5, S D=18.3$; $t(35)=0.63, p=.535)$. Also consistent with the properties of life scripts listed earlier, the standard deviations of expected age were smaller in positive categories $(M=2.71, S D=$ 2.06) than the standard deviations of expected age in negative categories $(M=10.08, S D=4.89 ; t(35)=6.45, p<.001)$. Only 4 of 29 positive categories had a standard deviation of age larger than 5 , while 7 of the 8 negative categories had a standard deviation larger than 5 .

\section{Effects of age}

The results of the participants were divided into three age groups (16-35, 36-55, 56-75 years). All three age groups mentioned many events from the life script: $86.4,89.5$ and $87.2 \%$. There was no difference between the age groups in regard to the proportion of events from the life script $(p=.221)$. Six events were always among the seven most

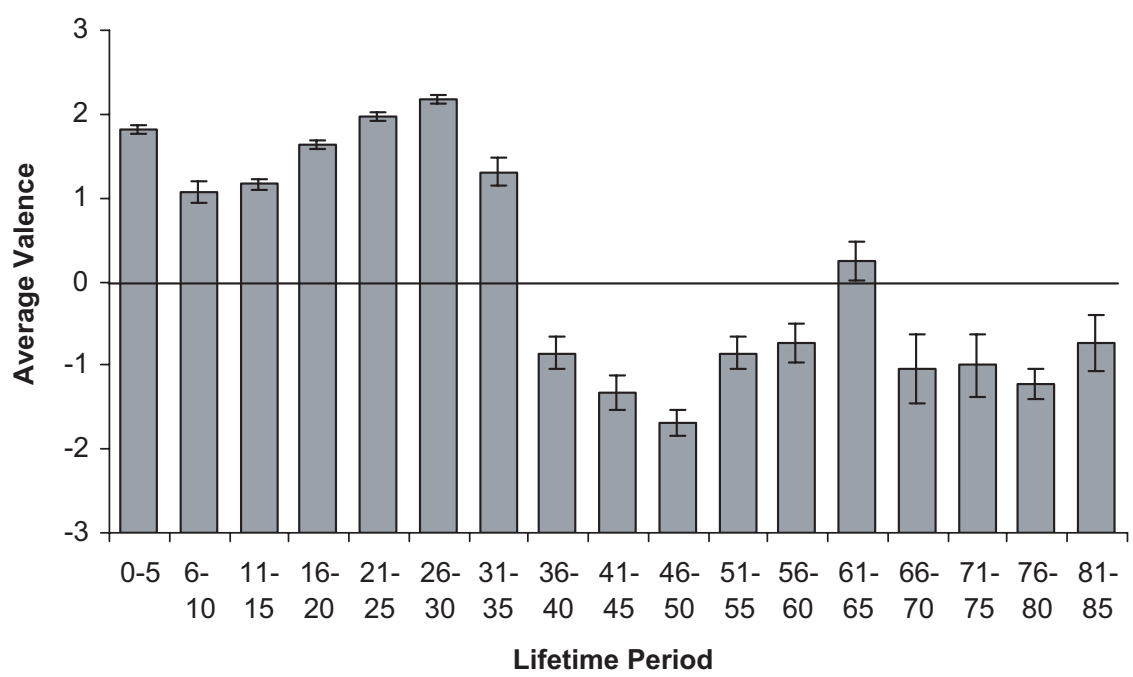

Figure 2. The average valence of the nominated events as a function of their expected age at occurrence 


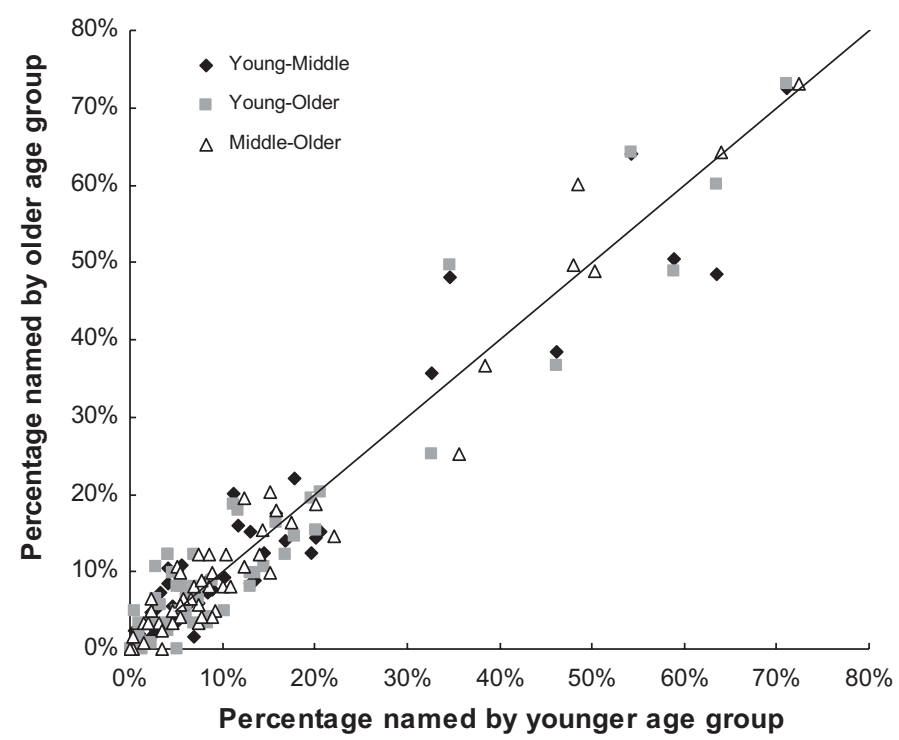

Figure 3. Comparison of the percentage of mentions per event category across the three age groups. On the $x$-axis the results of the younger age group from the comparison are given, while the results of the older age group are given on the $y$-axis

mentioned events: having children, begin school, marriage, falling in love, parents' death and first fulltime job.

The proportion of mentions of each event category was then compared between the three age groups (young and middle-aged adults, young and older adults, and middle-aged and older adults). In Figure 3, black diamonds represent categories with the percentage of mentions by young adults given on the $x$-axis and the percentage of mentions by middle-aged adults given on the $y$-axis. Grey squares depict the events with the percentages of mentions by young and older adults, while white triangles depict them with the percentages of mentions by middle-aged and older adults. Categories close to the diagonal line are events that had similar percentages for both age groups. As one can see from the event categories, the correlations between the proportions of mentions were extremely high (all $p \mathrm{~s}<.001$ ). The values of these correlations are given in upper half of Table 2, as are the correlations of the average expected age at occurrence, prevalence, importance and valence (all $p s<.001$ ). There were very high correlations for average expected age of occurrence and valence, but lower correlations for average prevalence and importance.

Participants of each age group were split into two equally sized groups and the correlation between these halves was calculated. Since the correlations within the age groups have fewer observations than the between-group comparisons,
Spearman-Brown prophesy formula (Brown, 1910; Spearman, 1910) was used to adjust the correlations. The withingroup comparisons, which are given in lower panel of Table 2, were similar to the correlations between the age groups, establishing that there were no age differences. If there would have been, then the split-half correlations should have been much larger. The lower values for the average prevalence and the average importance are therefore due to their limited reliability, which may be caused by the limited variability in these measures. The small standard deviations of importance and prevalence on trial level $(S D=1.04$ and 22.2) and category level $(S D=0.47$ and 16.9) support this interpretation.

For each of the 37 life script categories, we conducted a one-way ANOVA for the effect of age group on our four measures of the expected age of occurrence, prevalence, importance and valence. To hold the overall $p$-level at .05 for the 37 ANOVAs done on each of these dependent measures, we used a Bonferroni correction $(p=.05 / 37=.0014)$. None of the 148 ANOVAs was significant.

\section{Effects of education}

Similar analyses were conducted for the level of education, by dividing the results of the participants into four groups. The percentage of events from the life script were, respectively,

Table 2. The correlations of the frequency of mentions, average expected age of occurrence, average prevalence, average importance and average valence between and within young, middle-aged and older adults per category

\begin{tabular}{|c|c|c|c|c|c|}
\hline & Frequency & Age & Prevalence & Importance & Valence \\
\hline \multicolumn{6}{|c|}{ Correlations } \\
\hline Young-middle & .962 & .994 & .863 & 688 & .957 \\
\hline Young-older & .946 & .965 & .791 & .521 & .933 \\
\hline Middle-older & .978 & .974 & .875 & .538 & .972 \\
\hline \multicolumn{6}{|c|}{ Split-half correlations $\mathrm{s}^{\mathrm{a}}$} \\
\hline Young & .984 & .993 & .815 & 681 & .964 \\
\hline Middle & .984 & .991 & .950 & .572 & .969 \\
\hline Older & .984 & .980 & .830 & 689 & .915 \\
\hline
\end{tabular}

${ }^{a}$ Spearman-Brown prophesy formula was used to adjust for the lower number of participants in the split-half correlations. 
Table 3. The correlations of the frequency of mentions, average expected age of occurrence, average prevalence, average importance and average valence between and within participants with lower, middle, higher and scientific levels of education per category

\begin{tabular}{|c|c|c|c|c|c|}
\hline & Frequency & Age & Prevalence & Importance & Valence \\
\hline \multicolumn{6}{|c|}{ Correlations } \\
\hline Lower-middle & .938 & .954 & .534 & .516 & .788 \\
\hline Lower-scientific & .910 & .924 & .640 & .572 & .854 \\
\hline Middle-higher & .972 & .991 & .835 & .675 & .969 \\
\hline Middle-scientific & .976 & .987 & .796 & .774 & .957 \\
\hline \multicolumn{6}{|c|}{ Split-half correlations ${ }^{\mathrm{a}}$} \\
\hline Lower & .850 & .974 & .568 & .386 & .916 \\
\hline Middle & .981 & .987 & .723 & .780 & .918 \\
\hline Higher & .984 & .989 & .912 & .685 & .976 \\
\hline Scientific & .971 & .995 & .940 & .760 & .954 \\
\hline
\end{tabular}

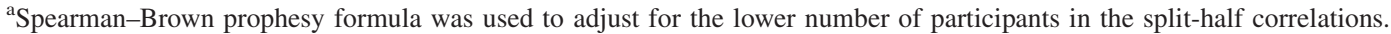

$90.0,85.7,88.0$ and $89.6 \%$ for the groups with a lower, middle, higher and scientific level of education. There was no difference between the groups in regard to the proportion of events from the life script $(p=.141)$. The same six events were constantly among the seven most mentioned categories.

Correlations between the frequencies with which the events were listed were extremely high. The values of these correlations are given in the upper half of Table 3. The correlations of the average expected age at occurrence, prevalence, importance and valence (all $p \mathrm{~s}<.001$ ), which are also given in the upper half, were high as well. The splithalf correlations for each level of education are included in the lower panel of Table 3 .

One-way ANOVAs were also conducted for the effects of education on the four measures of the expected age of occurrence, prevalence, importance and valence for each event category. Using a Bonferroni correction of $p=.0014$, only one of these 148 ANOVAs was significant: The valence measure for becoming a part of a group of friends, $F(3$, $30)=13.83, M S E=0.63, \eta_{p}^{2}=.580$, which was driven by the low education group having a negative mean, whereas the other groups had positive means.

\section{DISCUSSION}

Life scripts are culturally shared expectations about the timing of life events in an idealized life course (Berntsen \& Rubin, 2004). In this online study, participants were asked to imagine an ordinary infant. They were then asked to describe the seven most important events that were most likely to take place in the life of this prototypical child. The participants subsequently categorized the events, indicated the expected age of occurrence and the prevalence of the events, and rated the events on importance and valence. Nearly all participants in this study were female. It could therefore be the results here represent the cultural life script for Dutch women and that the life script for Dutch men is different, although Erdoğan et al. (2008) had found no gender effect in the Turkish life script.

Participants nominated more positive events than negative events. The positive events were as expected more likely to occur in early adulthood, while the negative events could happen at any moment in life. Because of this unequal distribution of positive and negative events, the events that were expected to occur after people turn 35 years old were rated on average to be negative. These results replicate earlier findings (Berntsen \& Rubin, 2004; Erdoğan et al., 2008; Rubin et al., 2009) and they are consistent with the assumptions that cultural life scripts favour positive events and events from early adulthood.

We examined whether the life script of young adults was identical to the life script of middle-aged and older adults. Although the older participants had lived through a larger part of their lives than younger participants, they shared the same cultural life script. All three age groups named begin school, fall in love, first fulltime job, marriage, having children and parents' death most frequently. Correlations of the number of mentions per event category between the age groups were extremely high and similar to the correlations within the age groups. The expected age of occurrence, prevalence, importance and valence per event category also did not differ between the age groups. Similar analyses were conducted for the effect of educational attainment. Correlations of the number of mentions between the groups with different levels of education were again extremely high and the expected age of occurrence did not differ either. Educational attainment also had no effect on prevalence, importance and valence, except for becoming a part of a group of friends. The results of the detailed analyses showed that the participants in our study shared the expectations about the timing of events in the life of a prototypical person, regardless of their age or level of education, suggesting that cultural life scripts are indeed semantic knowledge.

Cultural life scripts are used to retrieve autobiographical memories and to process life stories (Berntsen \& Rubin, 2004) and they can therefore be used to explain why people recall many events from the third decade of life when they are asked to recall the most important events from their lives (Rubin \& Schulkind, 1997). There is a large overlap between life script and the events that people recall when they are asked to give their life story (Rubin et al., 2009; Thomsen \& Berntsen, 2008), when they are asked to recall events which made them feel especially good about themselves (Collins et al., 2007), or when they are asked to name the most important events from their lives (Glück \& Bluck, 2007). The third decade is, however, not the time period from which 
people recall the most events when memories are cued with words (Rubin \& Schulkind, 1997). The temporal distribution of word-cued memories tends to peak in the second decade and this result should perhaps be attributed to other mechanisms (Janssen \& Murre, 2008; Rubin et al., 1998).

\section{ACKNOWLEDGEMENTS}

This study was supported by a Rubicon grant from the Netherlands Organization for Scientific Research (NWO) to SJ (Grant number: 446-06-031) and a grant from the National Institute of Mental Health to DR (R01-MH066079). We thank Jaap Murre and Martijn Meeter, the founders of the website on which the study was conducted.

\section{REFERENCES}

Berntsen, D., \& Jacobsen, A. S. (2008). Involuntary (spontaneous) mental time travel into the past and future. Consciousness \& Cognition, 17, 1093-1104.

Berntsen, D., \& Rubin, D. C. (2002). Emotionally charged autobiographical memories across the life span: The recall of happy, sad, traumatic, and involuntary memories. Psychology and Aging, 17, 636-652.

Berntsen, D., \& Rubin, D. C. (2004). Cultural life scripts structure recall from autobiographical memory. Memory \& Cognition, 32, 427-442.

Bohn, A. (in press). Generational differences in cultural life scripts and life story memories in younger and older adults. Applied Cognitive Psychology.

Bohn, A., \& Berntsen, D. (2008). Life story development in childhood: The development of life story abilities and the acquisition of cultural life scripts from late middle childhood to adolescence. Developmental Psychology, 44, 1135-1147.

Brown, W. (1910). Some experimental results in the correlation of mental abilities. British Journal of Psychology, 3, 296-322.

Collins, K. A., Pillemer, D. B., Ivcevic, Z., \& Gooze, R. A. (2007). Cultural scripts guide recall of intensely positive life events. Memory \& Cognition, $35,651-659$

Erdoğan, A., Baran, B., Avlar, B., Taş, A. Ç., \& Tekcan, A. İ. (2008). On the persistence of positive events in life scripts. Applied Cognitive Psychology, 22, 95-111.

Fivush, R., \& Nelson, K. (2004). Culture and language in the emergence of autobiographical memory. Psychological Science, 15, 573-577.

Glück, J., \& Bluck, S. (2007). Looking back across the life span: A life story account of the reminiscence bump. Memory \& Cognition, 35, 1928-1939.
Gosling, S. D., Vazire, S., Srivastava, S., \& John, O. P. (2004). Should we trust web-based studies? A comparative analysis of six preconceptions about Internet questionnaires. American Psychologist, 59, 93-104.

Habermas, T. (2007). How to tell a life: The development of the cultural concept of biography. Journal of Cognition and Development, 8, 1-31.

Habermas, T., \& Bluck, S. (2000). Getting a life: The emergence of the life story in adolescence. Psychological Bulletin, 126, 748-769.

Janssen, S. M. J., Chessa, A. G., \& Murre, J. M. J. (2007). Temporal distribution of favourite books, movies and records: Differential encoding and re-sampling. Memory, 15, 755-767.

Janssen, S. M. J., \& Murre, J. M. J. (2008). Reminiscence bump in autobiographical memory: Unexplained by novelty, emotionality, valence, or importance of personal events. Quarterly Journal of Experimental Psychology, 61, 1847-1860.

Janssen, S. M. J., Murre, J. M. J., \& Meeter, M. (2008). Reminiscence bump in memory for public events. European Journal of Cognitive Psychology, 20, 738-764

Neugarten, B. L., Moore, J. W., \& Lowe, J. C. (1965). Age norms, age constraints, and adult socialization. American Journal of Sociology, 70, $710-717$.

Reips, U.-D. (2000). The Web experiment method: Advantages, disadvantages, and solutions. In M. H. Birnbaum (Ed.), Psychological experiments on the Internet (pp. 89-114). San Diego, CA: Academic Press.

Reips, U.-D. (2002). Standards for Internet-based experimenting. Experimental Psychology, 49, 243-256.

Rubin, D. C., \& Berntsen, D. (2003). Life scripts help to maintain autobiographical memories of highly positive, but not highly negative, events. Memory \& Cognition, 31, 1-14.

Rubin, D. C., Berntsen, D., \& Hutson, M. (2009). The normative and the personal life: Individual differences in life scripts and life story events among U.S.A. and Danish undergraduates. Memory, 17, 54-68.

Rubin, D. C., Rahhal, T. A., \& Poon, L. W. (1998). Things learned in early adulthood are remembered best. Memory \& Cognition, 26, 3-19.

Rubin, D. C., \& Schulkind, M. D. (1997). Distribution of important and word-cued autobiographical memories in 20-, 35-, and 70-year-old adults. Psychology and Aging, 12, 524-535.

Rubin, D. C., Wetzler, S. E., \& Nebes, R. D. (1986). Autobiographical memory across the adult lifespan. In D. C. Rubin (Ed.), Autobiographical memory (pp. 202-221). New York: Cambridge University Press.

Rybash, J. M. (1999). Aging and autobiographical memory: The long and bumpy road. Journal of Adult Development, 6, 1-10.

Schank, R. C., \& Abelson, R. P. (1977). Scripts, plan, and knowledge. In P. N. Johnson-Laird, \& P. C. Wason (Eds.), Thinking. Readings in cognitive science (pp. 421-435). Cambridge: Cambridge University Press.

Spearman, C. E. (1910). Correlation calculated from faulty data. British Journal of Psychology, 3, 271-295.

Thomsen, D. K., \& Berntsen, D. (2008). The cultural life script and life story chapters contribute to the reminiscence bump. Memory, 16, 420-435. 